\title{
Optic nerve sheath fenestration in cryptococcal meningitis
}

\author{
Tatyana Milman' \\ Neena Mirani',2 \\ Roger E Turbin' \\ 'Ophthalmology Department, Institute \\ of Ophthalmology and Visual Science, \\ ${ }^{2}$ Pathology Department, University \\ Hospital, New Jersey Medical School, \\ Newark, NJ, USA
}

\begin{abstract}
A patient with acquired immunodeficiency syndrome (AIDS) developed crytpococcal meningitis, complicated by papilledema and severe progressive visual loss despite medical therapy. Bilateral optic sheath fenestration resulted in significant improvement in vision and resolution of papilledema. Histopathologic evaluation of the optic nerve sheath demonstrated numerous cryptococci. Optic nerve sheath fenestration may be an effective treatment method when high intracranial pressure is contributing to visual loss, even in the presence of involvement of the optic nerve sheath by the fungus.
\end{abstract}

Keywords: optic nerve sheath, cryptococcal, meningitis, fenestration

\section{Introduction}

Visual loss in cryptococcal meningitis may occur from chronically elevated intracranial pressure, adhesive arachnoiditis, or direct invasion of the visual sensory pathway by the organism (Kupfer and McCrane 1974; Ofner and Baker 1987; Tan 1988; Lipson et al 1989; Garrity et al 1993). Medical therapy with antifungal agents and surgical treatment, directed towards lowering the increased intracranial pressure, have met with variable success. There are, however, several reports of successful treatment of papilledema and visual loss by surgical methods, even in the presence of direct invasion of the brain tissues by the organism (Garrity et al 1993; Tan 1988). Our patient similarly experienced a remarkable improvement in vision and papilledema after optic nerve sheath fenestration despite the presence of cryptococcus in the optic nerve sheath.

\section{Case report}

A 25-year-old patient with acquired immunodeficiency syndrome (AIDS) developed headaches, seizures, altered mental status, and visual loss. Lumbar puncture showed markedly increased opening cerebral spinal fluid (CSF) pressure; cryptococcal organisms were identified by India ink preparation and in culture. Despite medical treatment with intravenous amphotericin B and oral fluconazole for cryptococcal meningitis, visual loss progressed to 20/200 OD and light perception OS within several weeks. Ophthalmoscopy revealed bilateral optic nerve edema (Figure 1A). Magnetic resonance imaging showed leptomeningeal and optic nerve enhancement without hydrocephalus (Figure 2). Because of progressive severe visual loss despite medical management, and in light of increased intracranial pressure and papilledema, the patient was recommended to have bilateral optic nerve sheath fenestration.

Histopathologic evaluation of the (bilateral) optic nerve sheath fenestration specimen revealed infiltration of the meninges by yeast-form organisms, consistent with cryptococci (Figure 3). Two weeks after surgery, the patient's optic nerve edema resolved (Figure 1B) and vision improved to 20/80 OD and hand motions OS. 


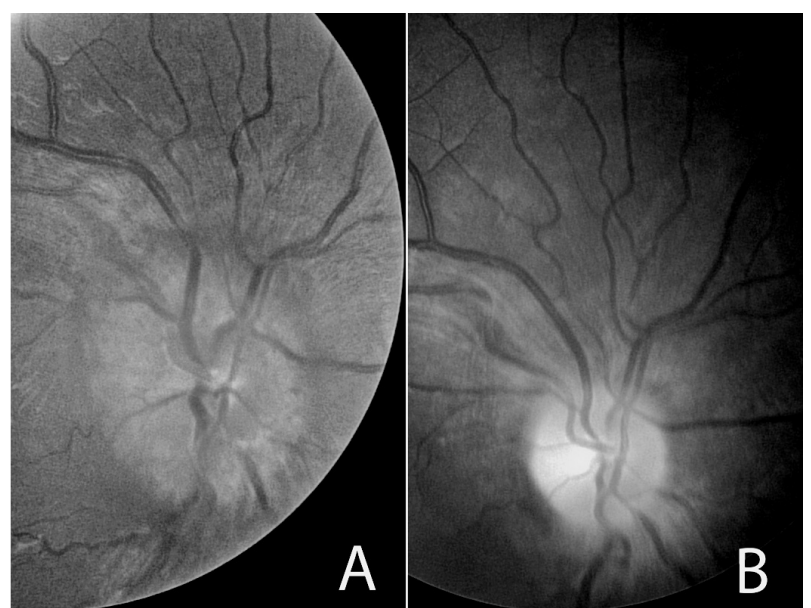

Figure I Fundus photograph of the right eye $(\mathbf{A})$ reveals marked optic nerve head edema. Same eye, two weeks after optic nerve sheath fenestration with resolution of optic nerve head edema (B).

\section{Discussion}

Cryptococcus neoformans is the most common life-threatening fungal pathogen that infects patients with AIDS (Garrity et al 1993). Cryptococcal infection typically manifests as meningitis with secondary ocular involvement. Abnormal ocular findings are seen in about $40 \%$ of the patients, with papilledema being the most common presenting sign (Lipson et al 1989).

The diagnosis of cryptococcal meningitis is suggested by a constellation of clinical and radiographic findings and is supported by a diagnostic lumbar puncture. High opening pressure and the presence of cryptococcal antigen and organisms in cerebrospinal fluid of affected patients are diagnostic

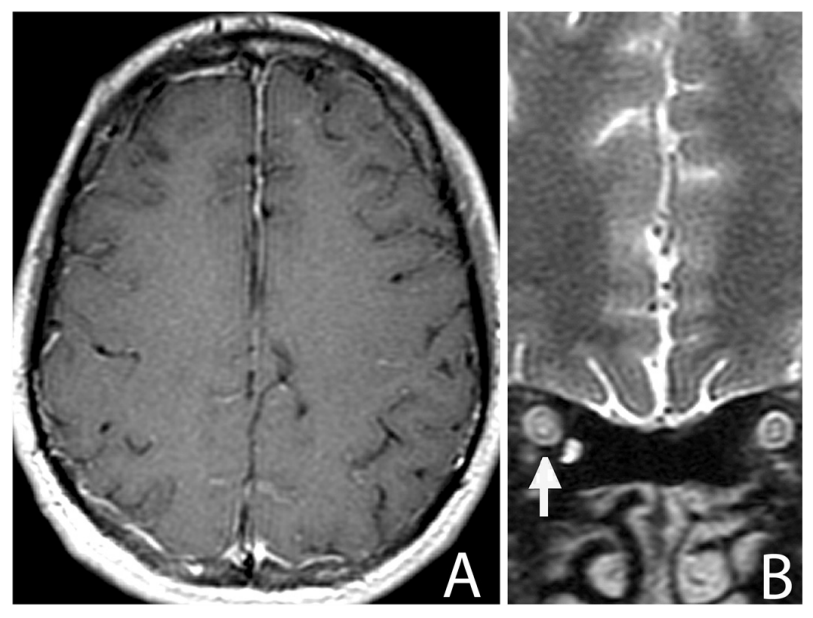

Figure $2 \mathrm{TI}$ weighted, axial MRI of the brain with contrast reveals diffuse leptomeningeal enhancement (A).T2 weighted, coronal MRI of the brain reveals thickening of the optic nerve-meningeal complex $(\uparrow)$ and increased CSF signal in subarachnoid space (B). Abbreviations: CSF, cerebral spinal fluid; $M R I$, magnetic resonance image.

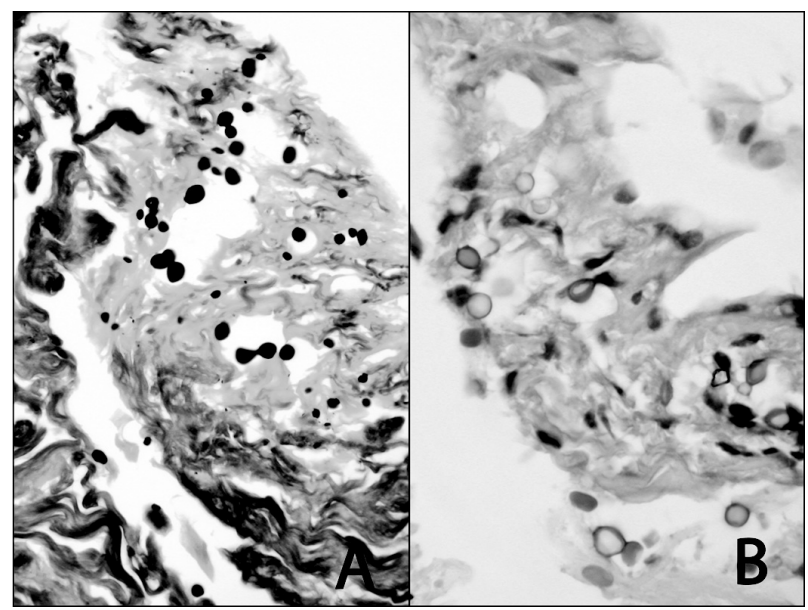

Figure 3 Budding yeast organisms stain with Gomori methenamine silver stain (A) (GMS stain; high power). The capsules of cryptococci stain with mucicarmine stain (B) (Mucicarmine stain; high power).

of cryptococcal meningitis (Ofner and Baker 1987; Tan 1988; Lipson et al 1989; Garrity et al 1993).

When the tissue is available for histopathologic diagnosis, cryptococcal organisms may be identified in meningeal sheath, subarachnoid space, and, occasionally, within the brain parenchyma. This budding yeast has thick gelatinous capsule that stain with periodic acid-Schiff, silver, mucicarmine, and acid mucopolysaccharide stains. Little reactive inflammation is seen in surrounding tissues (Ofner and Baker 1987; Tan 1988; Garrity et al 1993).

Untreated, cryptococcal meningitis is almost always a fatal disease. Medical treatment with intravenous amphotericin B and oral fluconazole has a guarded prognosis, and permanent neurologic and ophthalmologic sequelae are common (Tan 1988). Another treatment goal is directed toward lowering increased intracranial pressure, which may contribute to optic atrophy and visual loss. Repeated lumbar punctures, CSF diverting procedures, and optic nerve sheath fenestration have met with variable success (Ofner and Baker 1987; Tan 1988; Garrity et al 1993; Ferreira et al 1997). Interestingly, involvement of the brain by cryptococcal organisms has been demonstrated in several patients who benefited from a shunting procedure (Tan 1988). Garrity and associates (1993) similarly reported visual improvement in two patients with cryptococcal meningitis, treated with optic nerve sheath fenestration. Cryptococcal organisms were present in dural sheath specimens of both patients despite ongoing therapy with antifungal medication (Garrity et al 1993). Our patient had analogous presentation of decreased vision, optic nerve head edema, and increased intracranial pressure. Partial visual rehabilitation was achieved following optic nerve 
sheath fenestration despite histopathologically documented optic nerve sheath involvement by fungi.

It appears that no single formula can be applied in management of cryptococcal meningitis. Medical treatment of the infection should be aggressively pursued. When high intracranial pressure appears to contribute to visual loss, optic nerve sheath fenestration may offer a treatment alternative to protect the optic nerves from the effects of chronic papilledema.

\section{Disclosure}

This work was supported, in part, by an unrestricted grant from the Research to Prevent Blindness, Inc. NY, New York; Fund for the New Jersey Blind, Newark, New Jersey; Lions Eye Research Foundation of New Jersey, Newark, NJ;
The Eye Institute of New Jersey, Newark, New Jersey; and the Gene C. Coppa Memorial Fund, Newark, New Jersey. There are no conflicts of interest to report.

\section{References}

Ofner S, Baker RS. 1987. Visual loss in cryptococcal meningitis. J Clin Neuroophthalmol, 7:45-8.

Garrity JA, Herman DC, Imes R, et al. 1993. Optic nerve sheath decompression for visual loss in patients with acquired immunodeficiency syndrome and cryptococcal meningitis with papilledema. Am J Ophthalmol, 116:472-8.

Tan, CT. 1988. Intracranial hypertension causing visual failure in cryptococcus meningitis. J Neurol Neurosurg Psychiatry, 51:944-6.

Lipson BK, Freeman WR, Beniz, et al. 1989. Optic neuropathy associated with cryptococcal arachnoiditis in AIDS patients. Am J Ophthalmol, 107:523-7.

Kupfer C, McCrane E. 1974. A possible cause of decreased vision in cryptococcal meningitis. Invest Ophthalmol Vis Sci, 13:801-4.

Ferreira RC, Phan G, Bateman BJ. 1997. Favorable visual outcome in cryptococcal meningitis. Am J Ophthalmol, 124:558-60. 
